\title{
BMJ Open Effect of nutrition interventions on diet- related and health outcomes of Aboriginal and Torres Strait Islander Australians: a systematic review
}

\author{
Josephine Gwynn, ${ }^{1,2}$ Kyra Sim, ${ }^{2,3}$ Tania Searle, ${ }^{4}$ Alistair Senior, ${ }^{2,5}$ Amanda Lee, ${ }^{6}$ \\ Julie Brimblecombe ${ }^{7}$
}

To cite: Gwynn J, Sim K, Searle T, et al. Effect of nutrition interventions on dietrelated and health outcomes of Aboriginal and Torres Strait Islander Australians: a systematic review. BMJ Open 2019;9:e025291. doi:10.1136/ bmjopen-2018-025291

- Prepublication history and additional material for this paper are available online. To view these files, please visit the journal online (http://dx.do org/10.1136/bmjopen-2018025291).

Received 11 July 2018 Revised 14 December 2018 Accepted 17 December 2018

D Check for updates

(c) Author(s) (or their employer(s)) 2019. Re-use permitted under CC BY-NC. No commercial re-use. See rights and permissions. Published by BMJ.

For numbered affiliations see end of article.

Correspondence to Dr Josephine Gwynn; josephine.gwynn@sydney. edu.au

\section{ABSTRACT}

Objective To review the literature on nutrition interventions and identify which work to improve dietrelated and health outcomes in Australian Aboriginal and Torres Strait Islander people.

Study design Systematic review of peer-reviewed literature.

Data sources MEDLINE, PubMed, Embase, Science Direct, CINAHL, Informit, PsychInfo and Cochrane Library, Australian Indigenous Health InfoNet.

Study selection Peer-reviewed article describing an original study; published in English prior to December 2017; inclusion of one or more of the following outcome measures: nutritional status, food/dietary/nutrient intake, diet-related biomedical markers, anthropometric or health measures; and conducted with Australian Aboriginal and Torres Strait Islander people.

Data extraction and synthesis Two independent reviewers extracted data and applied the Quality Assessment Tool for Quantitative Studies from the Effective Public Health Practice Project. A purpose designed tool assessed community engagement in research, and a framework was applied to interventions to report a score based on numbers of settings and strategies. Heterogeneity of studies precluded a metaanalysis. The effect size of health outcome results were estimated and presented as forest plots.

Results Thirty-five articles (26 studies) met inclusion criteria; two rated moderate in quality; 12 described cohort designs; 18 described interventions in remote/very remote communities; none focused solely on urban communities; and 11 reported moderate or strong community engagement. Six intervention types were identified. Statistically significant improvements were reported in 14 studies of which eight reported improvements in biochemical/haematological markers and either anthropometric and/or diet-related outcomes. Conclusions Store-based intervention with community health promotion in very remote communities, fiscal strategies and nutrition education and promotion programmes show promise. Future dietary intervention studies must be rigorously evaluated, provide intervention implementation details explore scale up of programmes, include urban communities and consider a multisetting and strategy approach. Strong Aboriginal and Torres Strait Islander community engagement is essential for effective nutrition intervention research and evaluation.
Strengths and limitations of this study

- The first systematic review of dietary interventions for Australia's Aboriginal and Torres Strait Islander people.

- We provide a visual presentation of, and comparison among, the health outcome results of different studies by presenting these as forest plots.

- We developed and applied a novel method of assessing and reporting Aboriginal and Torres Strait Islander community engagement in research.

- Few appropriate quality assessment tools are available for assessment of public health interventions such as those reviewed in this paper.

- There is a potential for reporting bias where studies have been published in the grey literature or as reports and therefore not included according to our inclusion criteria.

\section{PROSPERO registration number CRD42015029551.}

\section{INTRODUCTION}

Indigenous people internationally frequently suffer greater early mortality rates and poorer health status when compared with non-Indigenous people. ${ }^{1}$ In Australia, the difference in mortality rates is among the highest worldwide, with life expectancy being 10 years younger for Aboriginal and Torres Strait Islander people (Australia's Indigenous people). ${ }^{12}$ Dietary factors alone contribute to overall disease burden at over three times the rate than non-Indigenous Australians. ${ }^{1}$ Diet-related chronic diseases including diabetes and cardiovascular disease are the major contributors to the substantial 'gap' in health. ${ }^{2}$ Aboriginal and Torres Strait Islander Australians are three and a half times more likely to suffer from diabetes and experience coronary heart disease (CHD) at younger ages and increased morbidity, with those in the age group of 35-44 years nearly five times 
more likely to suffer from CHD than non-Indigenous Australians. ${ }^{2}$ These and other significant and intransigent health issues requires more effective strategies, including dietary interventions, in order to reduce the health gap, yet the national 'Closing the Gap' strategy that aims to reduce the disadvantage experienced by Aboriginal and Torres Strait Islander Australians has been largely ineffective over a 10 -year period ${ }^{3}$ and does not include improved nutrition as a target. There have been calls for greater attention to nutrition and food security for Aboriginal and Torres Strait Islander Australians ${ }^{45}$; however, the 2018 Implementation Plan for the National Aboriginal and Torres Strait Islander Health Plan 2013-2023 includes few deliverables on nutrition. ${ }^{6}$

The causes of poor dietary intake among Aboriginal and Torres Strait Islander Australians are complex, have their origins in dispossession, marginalisation and extreme poverty and are compounded by ongoing socioeconomic, environmental and geographic factors. ${ }^{7}$ In general, few Australians meet dietary recommendations for healthy foods, ${ }^{8}$ and this is more pronounced among Aboriginal and Torres Strait Islander Australians with $41 \%$ of energy intake derived from discretionary foods and drinks (those high in saturated fat, added sugar and/or salt), compared with $35 \%$ of the energy intake of non-Indigenous Australians. ${ }^{9}$ On average, Aboriginal and Torres Strait Islander Australians consume $25 \%$ more free sugar per day than non-Indigenous Australians, with more derived from sugary drinks (67\% compared with $51 \%$ ); this difference is more marked among children. ${ }^{10}$

The only systematic review investigating dietary interventions with Aboriginal and Torres Strait Islander Australians limited its focus to assessing the effectiveness of nutrition education programmes on non-communicable diseases ${ }^{11}$ and included six studies, not all peer-reviewed. Other previous reviews $(n=4)$ on dietary interventions have been narrative in approach and were not confined to the peer-reviewed literature. ${ }^{5-12}$ All identified settings-based food supply policies and development of a dedicated Aboriginal and Torres Strait Islander nutrition workforce as effective strategies and most recommended a multistrategic approach to address broader determinants. ${ }^{12-14}$

We undertook a systematic review of the peer-reviewed literature that examined nutrition interventions aiming to improve diet-related and health outcomes in Australia's Aboriginal and Torres Strait Islander people.

\section{METHODS}

\section{Study selection process and eligibility criteria}

Articles were included in the review if they: described a nutrition intervention strategy and included one or more of the following outcome measures: nutritional status, food/dietary/nutrient intake, diet-related biochemical/haematological markers, anthropometric or health measures; were published in the peer-reviewed scientific literature, prior to/including December 2017 and in the English language; described an original study (not a review, report or study protocol); were conducted with Australian Aboriginal and Torres Strait Islander people; and included quantitative methods. Interventions that solely measured change in knowledge or attitudes, or reported on supplementation with nutrients, were excluded. The search strategy used is detailed in online supplementary table 1 .

Electronic databases were searched, as was the Australian Indigenous HealthInfoNet, ${ }^{15}$ and reference lists from assessed articles were hand-searched.

Articles were scanned and checked against the inclusion criteria, duplicate citations removed and those that met the inclusion criteria were read and assessed for inclusion, and their reference lists were manually checked for additional articles. Any disagreement about the eligibility of studies was resolved by consensus.

The Preferred Reporting Items for Systematic Reviews and Meta-Analyses checklist of items to include when reporting a systematic review was followed, and this review was registered with the PROSPERO register. ${ }^{16}$

\section{Quality assessment}

Articles were assessed for their quality using the Quality Assessment Tool for Quantitative Studies from the McMaster University Effective Public Health Practice Project (EPHPP) ${ }^{17}$ which includes six quality assessment components (selection bias, study design, confounders, blinding, data collection methods and withdrawals and dropouts). Each article was given a EPHPP Global Quality Assessment rating and rated weak if it scored two or more weak component ratings, moderate if it scored one weak rating or strong if it scored no weak ratings. The Australian National Health and Medical Research Council's evidence hierarchy for intervention research questions was also applied to each article. ${ }^{18}$ This evidence hierarchy is widely accepted among the scientific community and includes five levels ranked according to rigour from level 1 (systematic review of level 2 studies) to level 4 (case series with either post-test or pretest/post-test outcomes).

Articles were allocated for review by one of the authors; no reviewer was allocated an article that they had coauthored. For each article, two reviewers independently assessed quality, and any discrepancies in component ratings were resolved through discussion between reviewers and then the expert opinion of the group if required.

\section{Data extraction}

General characteristics of the article, the population, outcomes and measures were extracted. We applied the Index of Relative Indigenous Socioeconomic Outcomes to classify socioeconomic disadvantage of the study target population. ${ }^{19}$ Each study location was also assigned an Australian Standard Geographical Classification Remoteness Area classification. ${ }^{20}$ All reviewers independently checked the extracted data. Discrepancies were resolved by consensus. 
The principles of community engagement and governance as well as capacity building have been identified previously as critical to the conduct and outcomes of research with Aboriginal and Torres Strait Islander Australians ${ }^{21}{ }^{22}$ and were assessed in our review. Four key features that capture information about these principles were identified from key literature in this field by JB and $\mathrm{JG},{ }^{13}{ }^{21-26}$ applied to the final studies included in this review and referred to collectively as Community Engagement Intensity (CEI). Each study was assessed as either light ( $\leq 1$ feature), moderate (2-3 features) or strong (four features). An 'ecological approach' (multisetting and multistrategic addressing several targets including the individual) is necessary when implementing community-based interventions addressing a complex behaviour such as dietary intake ${ }^{27}$ is compatible with Aboriginal and Torres Strait Islander people's view of health and increasingly used in research and health policy work. ${ }^{21} 28{ }^{29}$ Each study was given an Ecological Approach Score ${ }^{29}$ (EAS) to enhance understanding of the scope and impact of the interventions; a score of 4 representing at least two strategy types and $\geq 3$ settings; lesser scores reflect fewer strategy types and settings.

\section{Health outcomes}

We quantitatively compared the results of health outcomes that were reported in four or more studies, these were: body mass index (BMI), blood glucose and triglycerides (TGs), total cholesterol (TC) and ratio of total to high-density lipoprotein cholesterol (TC:HDL-C). For outcomes of interest, we extracted the mean estimate before and after the intervention and associated SD and sample sizes. We calculated total TC:HDL-C ratios from reported mean values, with the SD for the ratio found by propagation assuming a weakly positive correlation (0.15) between HDL and TC at the individual level, as has been shown in the literature. ${ }^{30}$

For visual presentation of, and comparison among, the results of different studies, we estimated an effect size that corresponds to the change in the population mean biomarker following intervention. This was calculated as mean after intervention minus the mean at baseline; negative values correspond to the biomarker being lower after intervention. Many systematic reviews calculate effect sizes using a standardised mean difference such as Cohen's $d$; however, where data are reported in common and comparable units, as is the case here, it is preferable that differences are estimated on the raw scale, as they are easier to interpret. ${ }^{31}$ We calculated the $95 \%$ CIs for each change as $1.96 \times \mathrm{SE}$ of the difference. SEs of differences were found by propagation of error assuming a strong intraclass correlation (0.8), which is a strong correlation between repeated measures from the same individuals. $^{32} 33$

Where studies reported data from multiple time points postintervention, we used data from the most recent time point recorded (ie, the longest follow-up period) to maximise inference about the long-term impacts of the intervention (follow-up periods are noted throughout). Where studies reported their results in a stratified way (eg, by age and/or gender), we estimated the overall mean, as the average of stratified means weighted by the sample size in each subgroup.

\section{Patient and public involvement}

This paper was reviewed by the Aboriginal Health and Medical Research Council of New South Wales [AHMRC NSW]) prior to publication. The AHMRC NSW is the peak body representing Aboriginal and Torres Strait Islander Community Controlled Health Organisations in the state of New South Wales. No patients were directly involved in the development and conduct of the review.

\section{RESULTS}

\section{Study selection and characteristics}

The initial search yielded 1101 records, duplicates were removed and 50 eligible articles remained of which 35 met the inclusion/exclusion criteria for this review. The first article was published in $1980^{34}$ and the latest in $2017 .^{35}$ Figure 1 outlines the flow of included articles, table 1 summarises study characteristics and figure 2 presents health outcomes as forest plots. Online supplementary table 2 provides full details for each article.

The final 35 articles were derived from 26 studies and grouped according to their study of origin.

\section{Quality assessment}

Two ${ }^{36} 37$ of the 26 studies in this review were assessed as moderate with the rest assessed as weak (online supplementary table 3). Six studies ${ }^{38-43}$ included articles describing additional impact evaluations that were either 'nested'44-49 or 'extensions, ${ }^{50} 51$ of the original study, and two ${ }^{4450}$ of these were assessed as moderate.

EPHPP component ratings were largely strong for data collection and moderate for study design, however were weak for confounders and blinding and moderate to weak for selection bias. When studies (and articles that were 'nested' or 'extensions') were classified according to the NHRMC levels of evidence table for intervention research, ${ }^{18} 11$ were classified as level IV evidence, 21 as III-3, three as III-2 [ $\left.{ }^{3438} 46\right]$ and one as III- $1 .{ }^{36}$

\section{Nutrition intervention types}

Six types of dietary intervention studies were identified: nutrition education and promotion programmes primarily aiming to improve nutrition and including a 'healthy lifestyle' programme component ( $\mathrm{n}=8$ studies); store-based intervention with community health promotion ( $\mathrm{n}=5$ studies); return to traditional diet ( $\mathrm{n}=3$ studies); fruit and vegetable subsidy ( $\mathrm{n}=2$ studies); store environment and/or policy ( $\mathrm{n}=7$ studies) that included store/ organisation/government policy, food price discounts and the effect of store manager on diet; and preschool meal programme ( $\mathrm{n}=1$ study). 


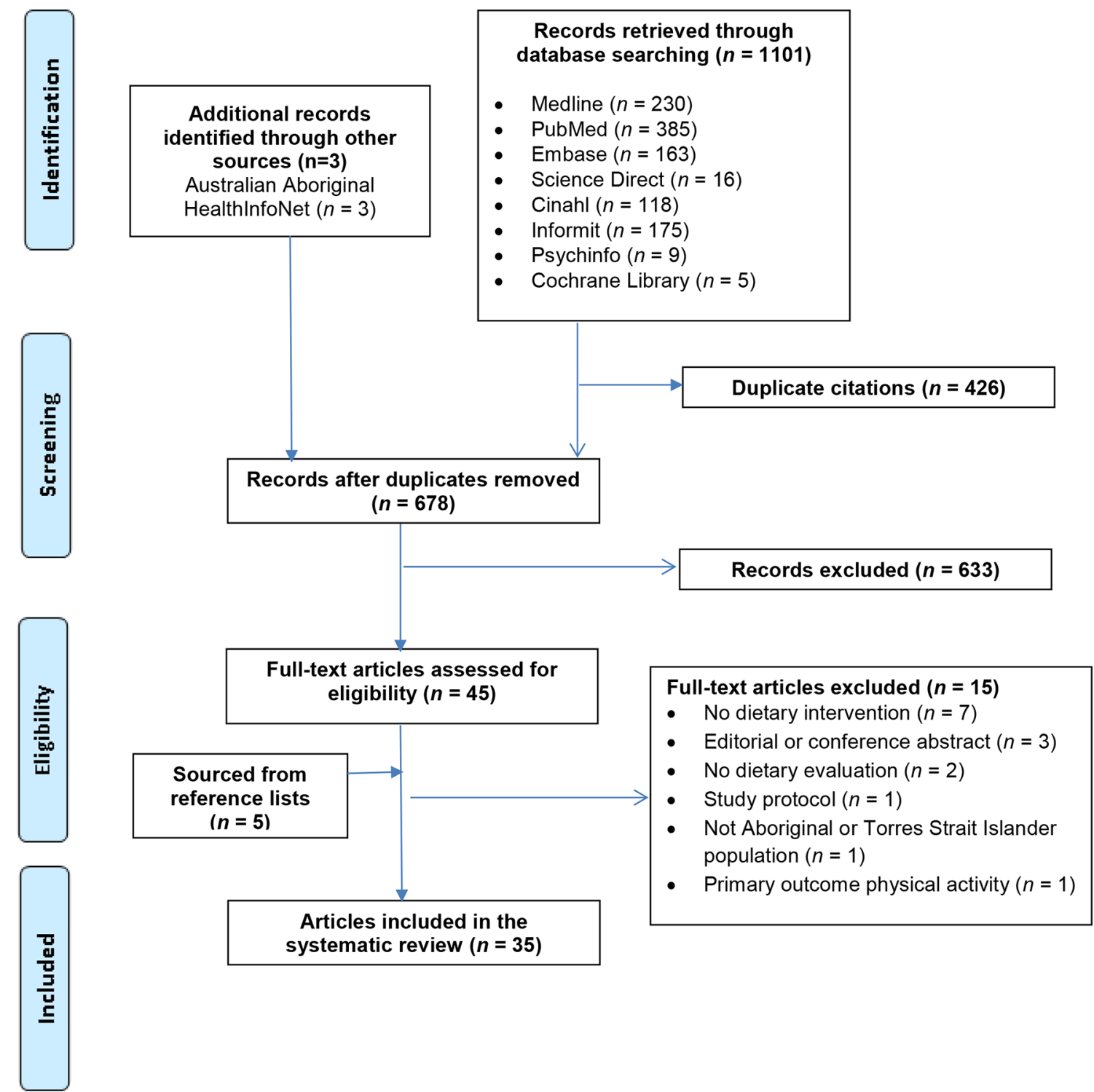

Figure 1 PRISMA flow chart of included and excluded articles. PRISMA, Preferred Reporting Items for Systematic Reviews and Meta-Analyses.

\section{Study locations}

The majority of the studies $(\mathrm{n}=18)$ included Aboriginal and Torres Strait Islander communities from areas classified as 'very remote'. ${ }^{20}$ Four studies included communities from areas classified as only 'inner' and/or' outer regional'. Only four studies ${ }^{34} 35253$ included participants from a major city and these studies also included other geographical locations.

\section{Study design}

This review includes 12 cohort studies, 1 cohort $^{39}$ with a 'nested' repeat cross-sectional study, ${ }^{44} 2$ repeat cross-sectional, ${ }^{40}{ }^{54} 2$ interrupted time series, ${ }^{55}{ }^{56}$ four case series ${ }^{53}{ }^{57-59}$ and one each of retrospective pre-post study, ${ }^{60}$ multisite case study, ${ }^{61}$ non-randomised controlled study, ${ }^{37}$ stepped-wedge randomised controlled trial ${ }^{36}$ and an interrupted time series with a control group ${ }^{38}$ and a 'nested' cohort. ${ }^{45}$

\section{Health outcomes}

Due to the relatively small number, and heterogeneity, of the studies, no meta-analysis was performed. There were insufficient common effect sizes to warrant aggregating the results. Outcomes reported in different articles arising from the one study were largely independent, other than in the Lee $e t a l^{88}$ study group (red blood cell folate at 12 months) ${ }^{45}$ and the O'Dea and Sinclair ${ }^{42}$ and O'Dea ${ }^{43}$ study groups (changes in weight, ${ }^{43}$ lipids $\left({ }^{424} 47-49\right]$ and diabetes markers ${ }^{47}$ ) where for completeness, we reported all results.

\section{Outcome measures}

Outcome measures of nutrition and health included the following categories: biochemical and/or haematological markers of dietary intake and/or health status $(n=12$ studies); food, diet and/or nutrient intake measures (24hours recall, survey and store-turnover/point-of-sale 

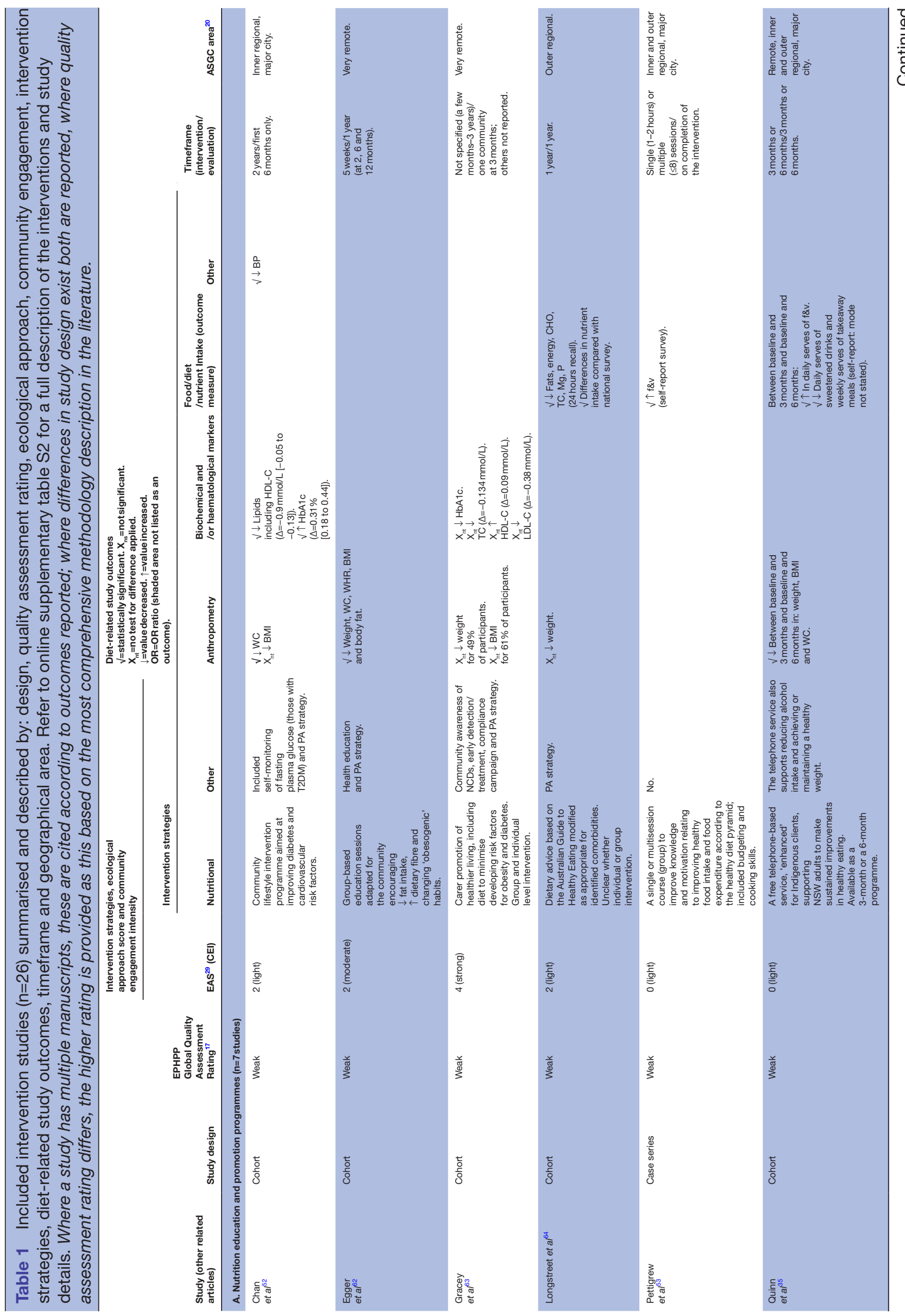

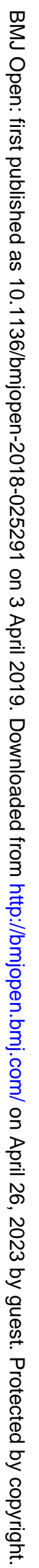




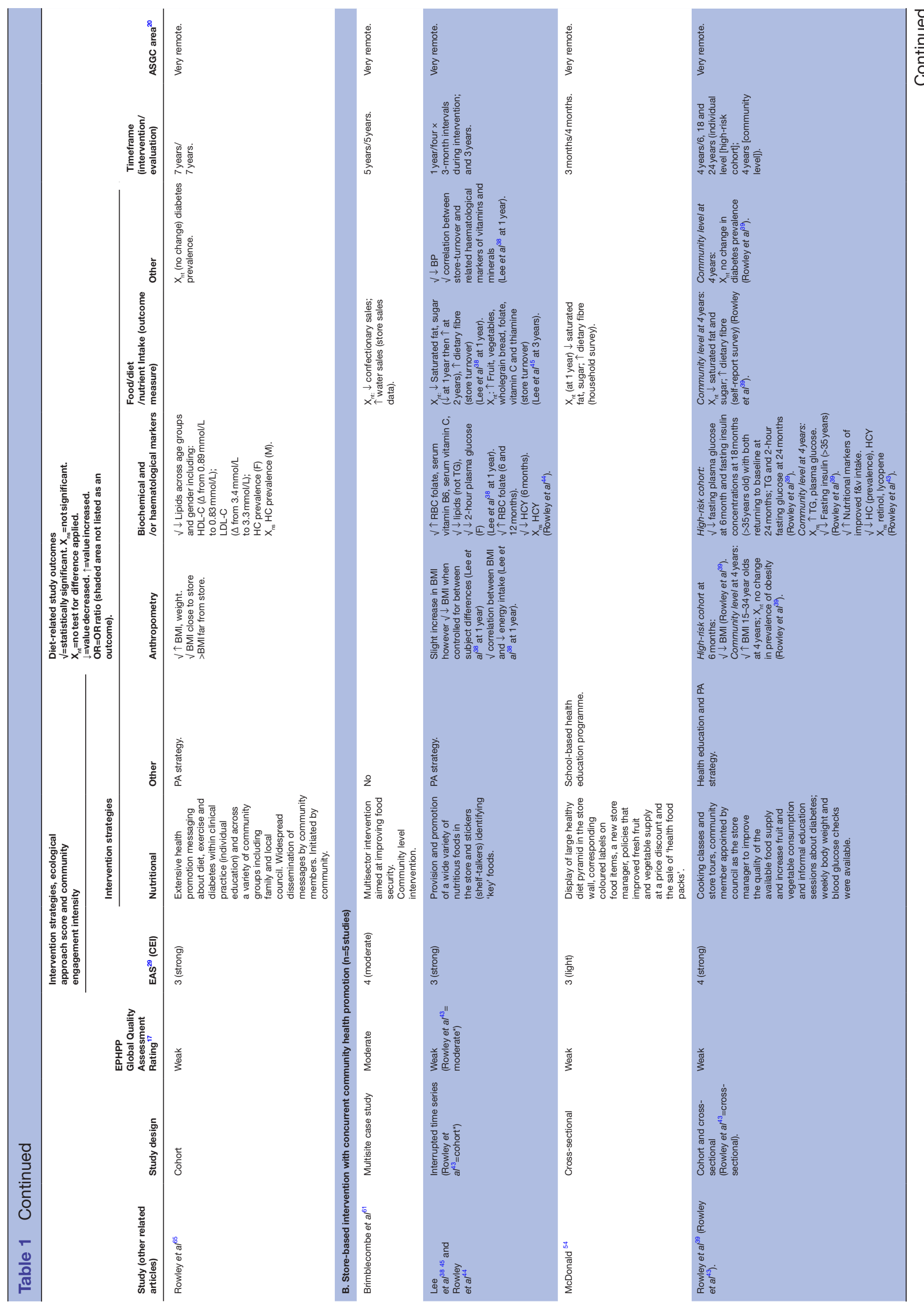

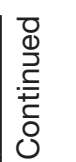

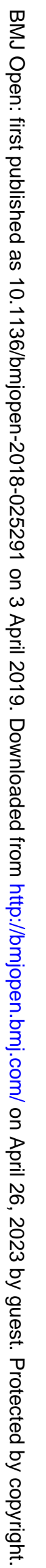




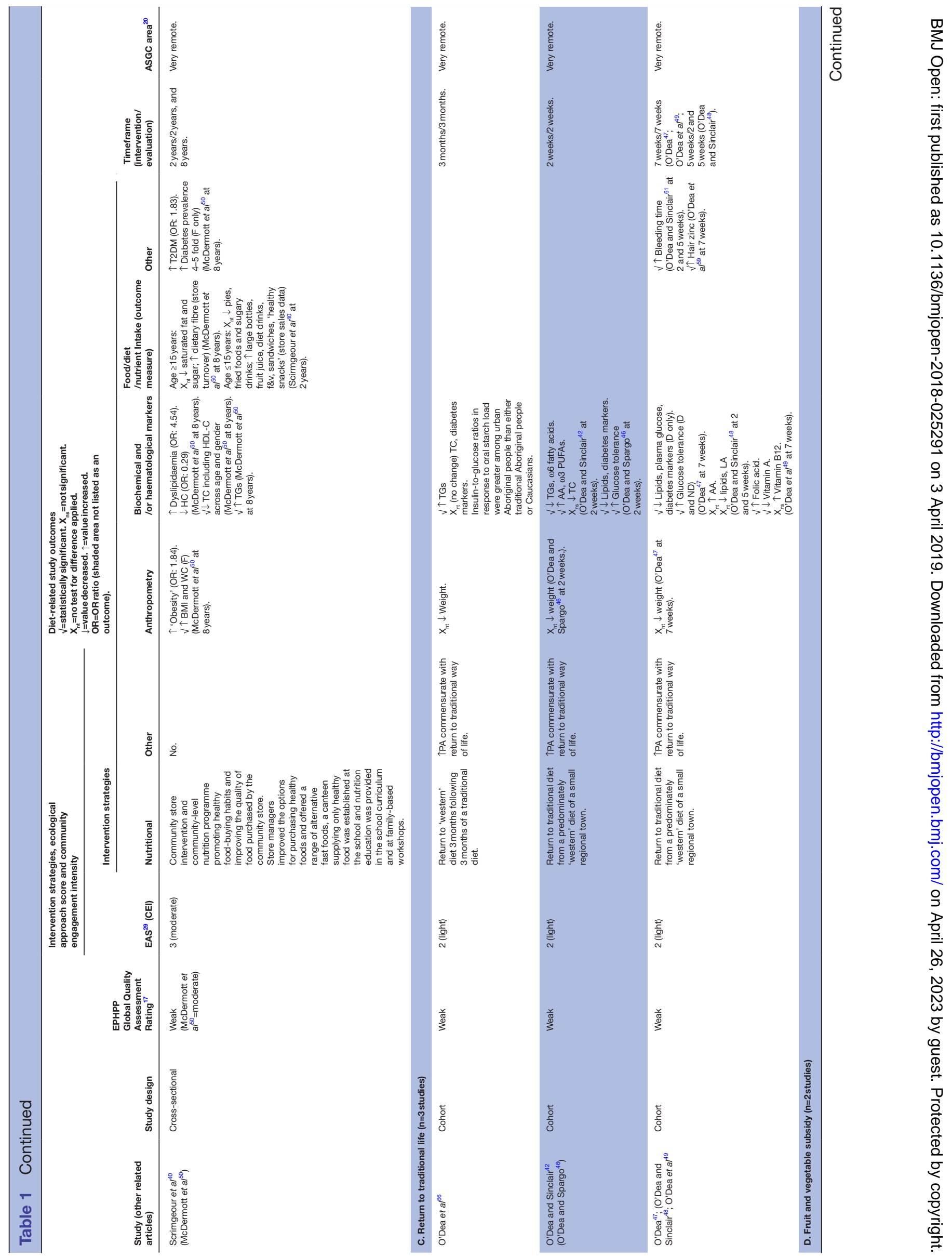




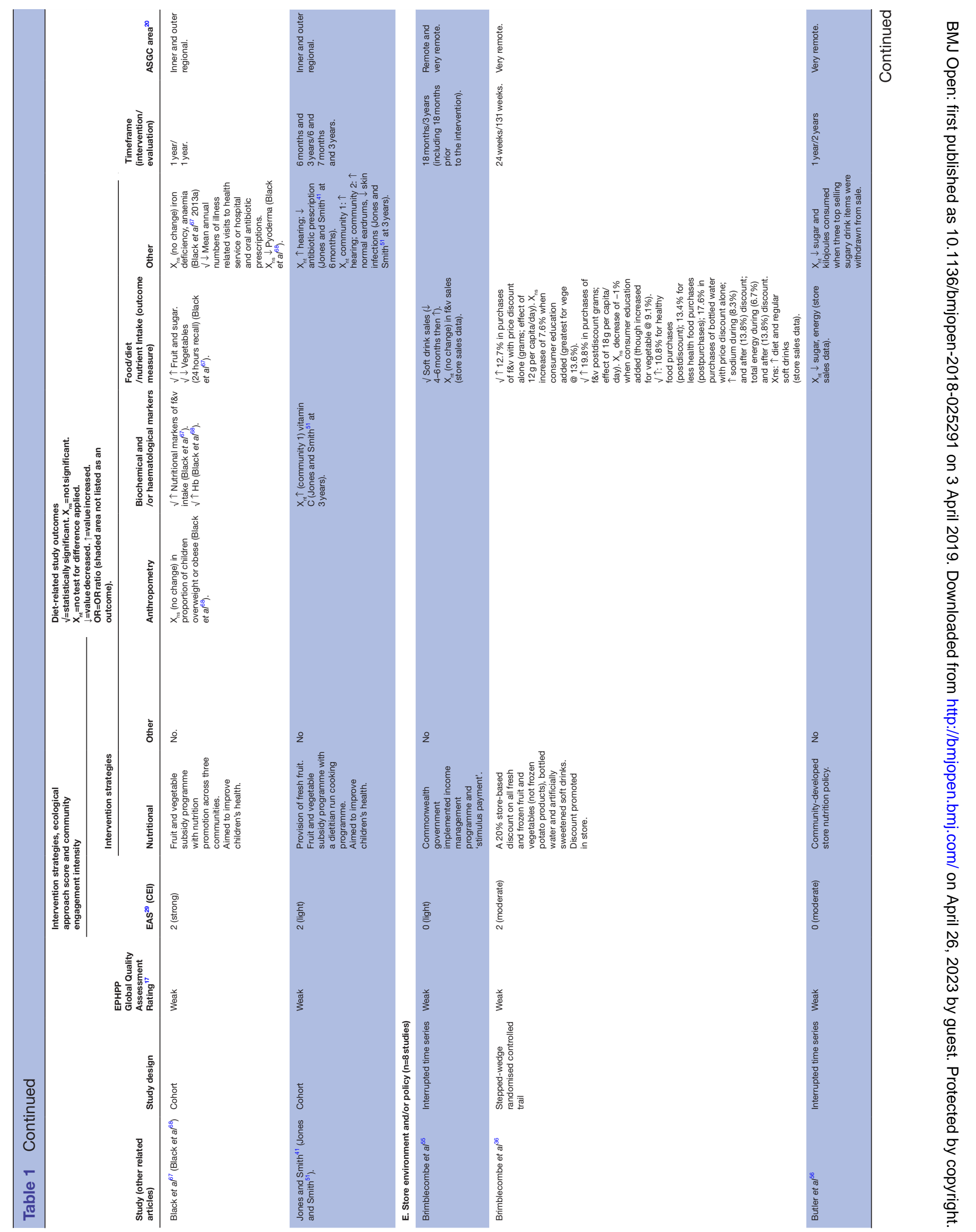




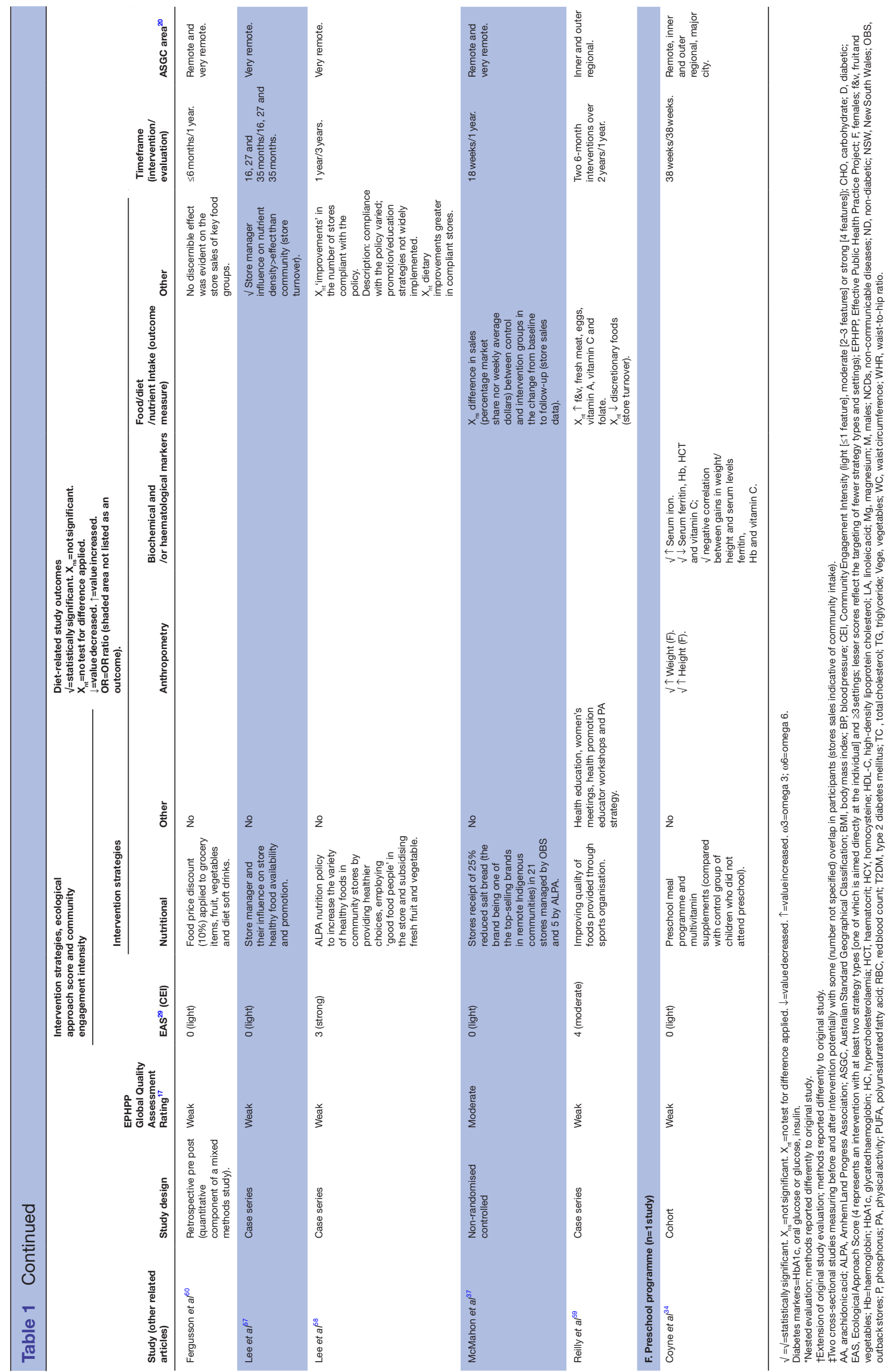

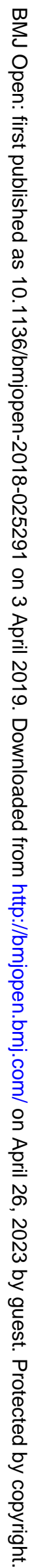



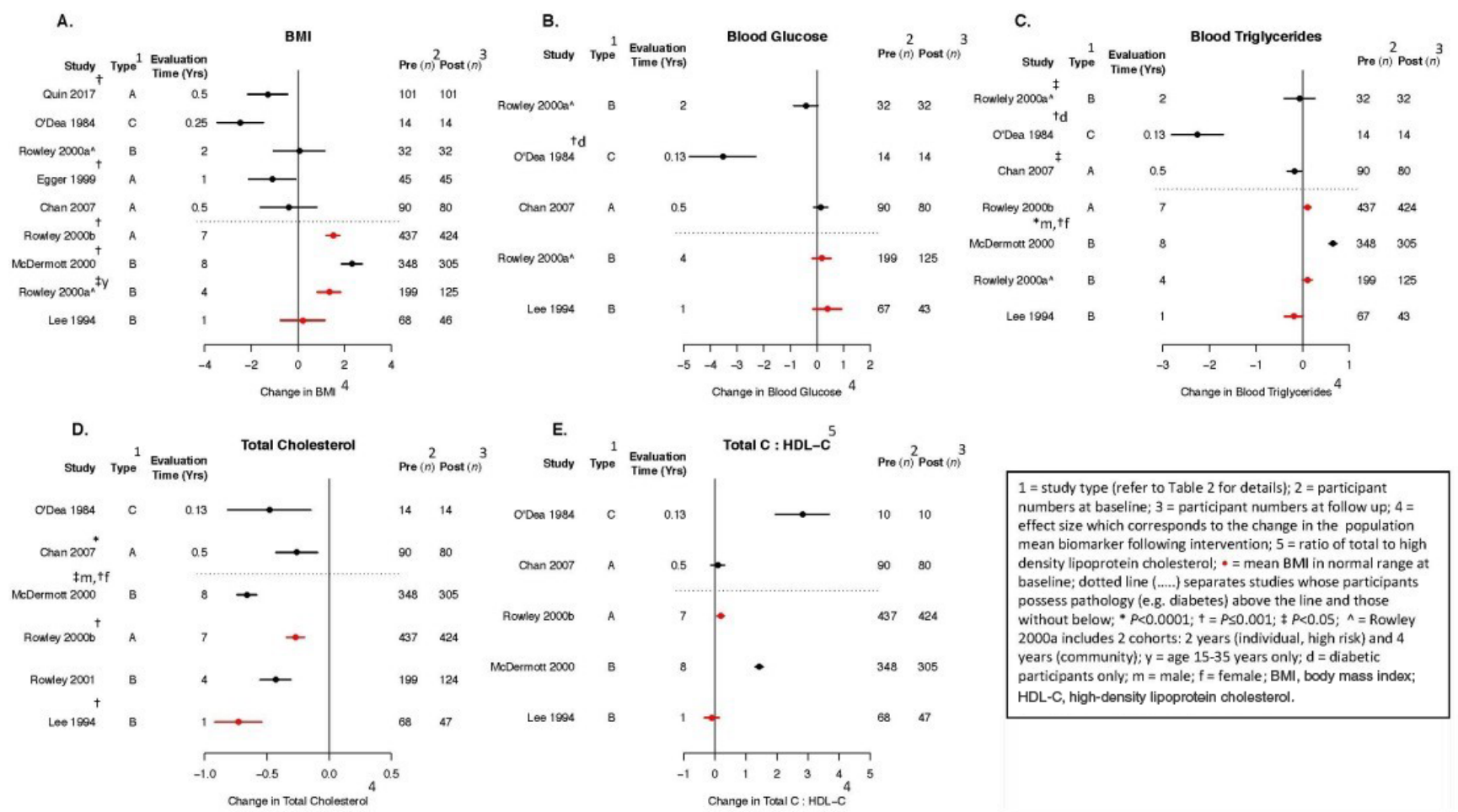

1 = study type (refer to Table 2 for details); 2 = participant numbers at baseline; $3=$ participant numbers at follow up; $4=$ effect size which corresponds to the change in the population mean biomarker following intervention; $5=$ ratio of total to high density lipoprotein cholesterol; $\boldsymbol{\bullet}=$ mean BMI in normal range at baseline; dotted line (.....) separates studies whose participants possess pathology (e.g. diabetes) above the line and those without below; $P<0.0001 ;{ }^{\uparrow}=P \leq 0.001 ; \uparrow P<0.05 ;{ }^{\wedge}=$ Rowley 2000a includes 2 cohorts: 2 years (individual, high risk) and 4 years (community); $y=$ age $15-35$ years only; $d=$ diabetic participants only; $m=$ male; $f$ = female; BMI, body mass index; HDL-C, high-density lipoprotein cholesterol.

Figure 2 Forest plots of health outcome data. Study types ${ }^{1}$ : nutrition education and promotion; store-based intervention with community health promotion; and return to traditional diet.

methods) ( $\mathrm{n}=14$ studies); anthropometric measures ( $n=14$ studies) and other outcomes ( $n=12$ studies). Fourteen studies included more than one outcome. We notate study outcomes in table 1 according to whether a test for significance was applied (and whether findings were significant), and if not, then all other findings (including descriptive) are reported.

Most of the studies that included a nutrition education and promotion component also included a physical activity component assessed as not being the primary focus of the study. Only two studies described a theoretical framework. ${ }^{5962}$

\section{Ecological approach and Aboriginal and Torres Strait Islander engagement and governance features}

Nine of the 26 studies attained the higher EAS of 3 or 4, indicating two or more settings with at least one intervention strategy aimed at the individual level plus at least one other (see table 1), and eight studies attained a score of 0 .

Aboriginal and Torres Strait Islander engagement and governance 'Key Features' are listed in online supplementary table 4, and studies reporting on these features were referenced. Around $50 \%$ of the studies attained moderate or strong CEI score. Sixty-eight per cent of studies reported including community governance though detail was scant. Fewer than $50 \%$ of studies did not report either providing results to the community $(40 \%)$ nor whether the study had been initiated by the community $(48 \%)$. Only $12 \%$ of studies reported providing a 'developmental pathway' to building Aboriginal and Torres Strait Islander research and programme delivery capacity, with most effort $(50 \%)$ directed towards shorter term 'learning opportunity and skill development'.

\section{Outcomes by intervention type}

Nutrition education and promotion programmes

Seven studies described this type of intervention, $35525362-65$ and all were assessed as weak in quality. Six were cohort studies, and another a case series. ${ }^{53}$ Evaluation timeframes ranged from single education sessions to 2 years, and one at 7 years. ${ }^{65}$ Studies that included a range of geographical locations were usually conducted in group or community settings and two included children. ${ }^{63}$ CEI varied with two studies ${ }^{63}$ rated as 'strong' and possessing an EAS of 3 and 4 .

Six of the seven studies that tested for statistical significance showed positive outcomes for some measures, ${ }^{35} 5253626465$ including in two ${ }^{5265}$ of the three studies that measured biochemical/haematological plus anthropometric outcomes. Adverse outcomes were shown for mean glycated haemoglobin ${ }^{52}$ and mean HDL-C, 5265 and mean BMI increased in another at 7 years. ${ }^{65}$

Three studies reported on self-reported dietary intake and found associated statistically significant improvements in fruit and vegetable intake, ${ }^{35} 53$ energy intake (decreased) and some macronutrients/micronutrients. ${ }^{64}$

Store-based intervention with concurrent community health promotion

Five studies (nine articles) described this intervention type. ${ }^{38-40} 444545505461$ Two studies were assessed as cross-sectional $^{405054}$; one an interrupted time series with 
a control group ${ }^{38}$ (included a 'nested' cohort design $)^{45}$; one a multisite case study ${ }^{61}$; and one a cohort ${ }^{39}$ (included a 'nested' cross-sectional design). ${ }^{44}$ All were assessed as weak quality with two ${ }^{3840}$ including additional impact evaluations both assessed as moderate. ${ }^{45}{ }^{50}$ Evaluation timeframes were up to 8 years, CEI was moderate to strong in all but one study, ${ }^{54}$ all attained an EAS of 3 or 4 and were conducted in very remote locations.

Three of the four studies tested for statistical significance and found associated positive outcomes, ${ }^{38} 394445$ including for biochemical/haematological and anthropometric outcomes. Mean lipids/diabetes-related markers, ${ }^{38}{ }^{39}$ markers of improved fruit and vegetable intake (eg, $\beta$-carotene $)^{44}$ and homocysteine (HCY) ${ }^{44} 45$ showed associated improvements between 2 years and 4 years. HDL-C was only assessed in the study conducted over 8 years ${ }^{50}$ and decreased. BMI significantly declined in the short term (6 months and 1 year $)^{38} 39$ and significantly increased over the longer term (4 and 8years) ${ }^{39} 50$ Improvements in diet-related outcomes were reported in all studies; however, statistical significance was not assessed.

\section{Return to traditional way of life}

These three cohort studies (six articles) ${ }^{42} 4347-4966$ were conducted with remote Aboriginal and Torres Strait Islander adults (diabetic and non-diabetic) and published between 1980 and 1985. These were assessed as weak in quality, with light CEI, and reported on the impact of interventions of between 2 weeks and 3 months.

Five articles ${ }^{42} 4347-49$ reported on evaluations up to 7 weeks while living a traditional life and consuming a traditional diet. Statistically significant improvements in TGs, very low-density lipoprotein cholesterol (VLDL-C) and diabetes markers were reported, along with a significant drop in HDL-C, and in one article noted to be present among those with diabetes only. ${ }^{49}$ Improvements were also reported for omega- 3 and omega- 6 fatty acids and nutritional markers. Three months after return to an 'urban' diet', a significant increase in TGs was found. ${ }^{66}$ While improvements in anthropometric outcomes (weight) were reported, statistical significance was not assessed.

\section{Fruit and vegetable subsidy}

Two studies (four articles) ${ }^{41} 516768$ included this intervention type and were derived from the continuation and evolution of the one nutrition programme initiated in 2005. ${ }^{41}$ Both were cohort design and participants were aged 17 years and under, and both were assessed as of weak quality. CEI was assessed as strong in the later study $^{6768}$ and both attained an EAS of 2.

The earlier study ${ }^{41}$ noted improvements in health (improvements in hearing); however, no tests for significance were applied. The later study ${ }^{6768}$ found statistically significant improvements in nutritional markers of fruit and vegetable intake and in fruit intake ${ }^{67}$ and health (decrease in illness-related visits to health service or hospital and oral antibiotic prescriptions) ${ }^{68}$ Adverse outcomes were that self-reported intakes of sugar increased and vegetables decreased. ${ }^{67}$

\section{Store environment and/or policy}

Eight studies described this intervention type $\mathrm{e}^{363^{35-60}}$; all but one ${ }^{59}$ were conducted in remote/very remote Aboriginal and Torres Strait Islander communities and included two studies assessed as moderate in quality. These studies differed in store environment strategy, and diverse study designs included: case series $(n=3)^{57-59}$; interrupted time series $(n=2)^{55} 56$; and one each of non-randomised controlled, $^{37}$ stepped wedge randomised controlled ${ }^{36}$ and retrospective pre-post. ${ }^{60}$ Four studies reported on the impact of the intervention at 2 years or more ${ }^{55-58}$ and all outcomes were assessed using the store turnover/ point of sale methods. Participants included all members of the communities, three studies applied an ecological approach $^{365658}$ and CEI varied.

Two studies found that a remote store manager supportive of healthy food provision $\left[{ }^{57}\right]$ and a store discount of $20 \%{ }^{36}$ were associated with a positive impact on community-level dietary-related outcomes. The latter study found a statistically significant increase in purchases of key foods including fruit, vegetables and bottled water; however, no impact on the purchase of diet and regular soft drinks. ${ }^{36}$

The introduction of a nutrition policy was associated with improvements in dietary-related outcomes in very remote communities. ${ }^{58}$ By contrast, a federal government-instigated income management strategy in very remote communities was associated with a statistically significant increase in soft drink sales and no change fruit and vegetables sales after 6 months. ${ }^{55}$ A price discount of $10 \%$ was associated with no impact on sales of grocery products, fresh fruit and vegetables and diet soft drinks. ${ }^{60}$

\section{Preschool meal programme}

This one cohort study implemented a preschool meal programme, provided multivitamin supplements and was conducted at a time when undernutrition and poor growth among Aboriginal and Torres Strait Islander children were common. The study was implemented across a range of geographical areas and demonstrated improvements in the height and weight of children. ${ }^{34}$

\section{Impact on health outcomes}

Studies from three intervention types (nutritional education and promotion, ${ }^{35} 526265$ store-based nutrition intervention with community health promotion or environment/policy ${ }^{38} 3950$ and return to traditional diet $^{43}$ ) were included in the forest plots (see figure 2). Decrease in BMI was only apparent in studies, which targeted those over the healthy weight range over a short time frame (ie, 3 months-1 year). ${ }^{35} 435262$ Small increases in BMI were apparent in four studies ${ }^{38-40} 65$ conducted in remote communities with three ${ }^{38} 3965$ possessing a mean BMI at baseline within normal range and at follow-up within or 
just over the normal range. Return to traditional diet over a short time frame demonstrated the most consistently positive impact on health outcomes, other than for TC:HDL-C, ${ }^{43}$ where a large decline in VLDL-C relative to HDL-C (decreased) and LDL-C (increased) was reported by the authors as a contributing factor to the change in distribution of TC. There was little impact on blood glucose or TG levels reported in other studies. TC declined for all studies regardless of the timeframe and whether the study was a 'targeted' intervention. However, TC:HDL-C increased in two of these studies ${ }^{43}{ }^{50}$ with another three demonstrating little change in ratio. ${ }^{38} 5265$ The study conducted over the longest time frame $(8 \text { years })^{50}$ noted a reversal of all positive health outcomes over time other than for TC.

\section{DISCUSSION}

This review of nutrition intervention studies that aimed to improve diet-related and health outcomes in Australian Aboriginal and Torres Strait Islander populations found statistically significant improvements in a range of outcomes across all intervention types and settings in the short to medium term. Store-based interventions, including a food price strategy, ${ }^{36}$ combined with community health promotion demonstrated most promise in very remote locations, ${ }^{36}$ 38-40 42444650 with all describing improvements in diet-related outcomes, although only one tested for statistical significance. ${ }^{36}$ Three of these six studies reported statistically significant improvements in some health outcome measures including $\mathrm{BMI}^{38} 39$ and biochemical/haematological markers of good nutrition and health $\left(\mathrm{TC}^{38}{ }^{50}\right.$ (over 8years); folate ${ }^{38}$; plasma glucose $\left.^{38}{ }^{39} ; \mathrm{HCY}^{38}{ }^{39} ; \mathrm{TG}^{38}{ }^{39}\right)$. In a regional area, the fruit and vegetable subsidy programme showed encouraging results with statistically significant improvements in children's biochemical/haematological markers of nutrition and health outcomes. ${ }^{6768}$ 'Nutrition education and health promotion programmes', mostly targeting populations with pre-existing health conditions, showed potential at the group education level across geographically diverse groups. ${ }^{52} 53626465$ Group nutrition education was also identified as a promising strategy in another review. ${ }^{11}$

Improvements reported in the store-based and community health promotion studies are likely related in part to their adoption of a strong ecological approach and moderate to strong community engagement in discrete communities, reflecting the strong evidence for approaches to addressing poor dietary intake which are both multisetting and multistrategy. We urge researchers to rigorously evaluate impact on diet-related outcomes to provide clear evidence of change. Store-based nutrition education (store tours and information labels on shelves) has also been identified as a promising strategy in another review. ${ }^{11}$ Food subsidy and price discount strategies should be further investigated for effect, feasibility and viability across Aboriginal and Torres Strait Islander communities regardless of geographical area. Economic analyses of such strategies are also needed as is examination on how best to 'scale-up' promising interventions to ensure viability for business and of cost-benefit to government along with benefit for the consumer. Lower discounts have demonstrated no discernible impact, ${ }^{60}$ and effect on the purchase of unhealthy foods with discount savings is yet to be fully understood as is the benefit of including an in-store strategy that seeks to deincentivise unhealthy food purchasing using retail marketing strategies. ${ }^{69} \mathrm{~A}$ recent cost-effectiveness study has found that a single strategy price discount on healthy foods may have negative health effects and that combining discounts with a comprehensive range of other strategies may be preferable. ${ }^{70}$ Income management strategies appear to have no positive effect on population level fruit, vegetables and soft drink sales. ${ }^{55}$ It must be noted that large changes in dietary intake are required to significantly impact on health outcomes, and this necessitates that the underlying determinants of such changes are simultaneously addressed.

Return to a traditional diet and lifestyle showed improvements in key health indicators over a very shorttime period ${ }^{42} 434^{47-49}$; however, these reversed once the participants returned to the less healthy 'urban' diet. ${ }^{66}$ One study noted that physical activity was 'generally high' (p. 32) ${ }^{66}$ and this may have also contributed to results, though not examined in the study. This seminal study demonstrated that Aboriginal and Torres Strait Islander Australians can experience dramatic health benefits from dietary improvement and in a very short time period and has inspired subsequent community-based studies. These studies have informed current dietary recommendations for Aboriginal and Torres Strait Islander Australians. ${ }^{7}$

The preschool meal programme conducted in 1980 found improvements among children who were underweight initially. Nutrition policies and programmes now exist in Australian preschool settings. ${ }^{69}$ Only two studies examined change in health outcomes over the longer term (7-8years), ${ }^{50} 65$ and these were not sustained despite small decreases in TC thought to reflect a commensurate fall in HDL-C. While maintaining positive health outcomes is a challenge also in similar studies conducted in the general population, ${ }^{71}$ additional barriers to healthy diet in Australian Aboriginal and Torres Strait Islander people exist ${ }^{72}$ including lower gross weekly income, ${ }^{73}$ the high cost and poor availability of healthy foods and increasing availability of unhealthy foods in many communities. ${ }^{74}$ Food insecurity is higher among Aboriginal and Torres Strait Islander Australians (22\%) than other Australians $(4 \%){ }^{8}$

The majority of studies $(n=17)$ scored the lower EAS of between 0 and 2 suggesting that multisetting and strategy interventions are yet to be fully embraced despite evidence to support this approach, possibly because of the significant resources and workforce capacity required to implement. ${ }^{28}$ One study in this review $^{61}$ describes a 5 -year project conducted with four remote communities and offers insights into establishing 
a multisectoral, multisetting strategy systems approach to dietary improvement. Studies with strong Aboriginal and Torres Strait Islander community engagement generally reported a wider range of outcomes than others with less intense engagement and tended to be associated with more comprehensive evaluation. This is likely a result of the relevance of the study to the community and trust between partners, reflecting key Australian guidelines on the conduct of research with Aboriginal and Torres Strait Islander communities. ${ }^{21}$ Building research capacity is recognised as an essential principle of research conducted with Aboriginal and Torres Strait Islander communities, ${ }^{26}$ is a requirement of ethical research conducted with communities, ${ }^{21}$ will help ensure high-quality research going forward and was not evident in most studies. The small proportion of studies reporting details of their community engagement and governance may be a factor of word limit restrictions imposed by journals and that these aspects of a study are yet to be accepted as integral to the report of methods.

The predominance of a weak overall quality rating for studies in this review is a result of generally weak scores for the assessment components of selection bias, presence of confounders and blinding. This may reflect brief or poor description of methods in the manuscripts and/ or challenges around the suitability of empirical research (including randomised controlled trials) in the small discrete 'real-world' Aboriginal and Torres Strait Islander communities where most studies were located.

\section{Implications and future directions}

Multisetting and strategy approaches that support Aboriginal and Torres Strait Islander leadership and include addressing the underlying determinants of dietary intake are strongly recommended to intervene successfully in the complex systems that surround access to healthy foods in Aboriginal and Torres Strait Islander communities. Strong Aboriginal and Torres Strait Islander community engagement and governance is central to comprehensive implementation and evaluation of dietary intervention programmes and to embedding successful approaches and policies within communities. In remote and very remote Aboriginal and Torres Strait Islander communities, a suite of approaches including store-based strategies that address healthy food availability and consider price discounts, with community-wide nutrition education and promotion programmes, should continue to be delivered and evaluated. Future studies need to explore the longterm feasibility, viability and impact of fiscal (subsidy and discount) strategies on diet and markers of good health across Aboriginal and Torres Strait Islander communities including urban settings. Impact on less healthy food purchases should also continue to be investigated and addressed. Particularly in the absence of adequate legislative support to improve food supply, the engagement of retailers and community store owners in design, implementation and evaluation is essential for sustainability and scale-up of such strategies. Heterogeneity of the interventions, settings and methods of studies included in this review, along with at times unclear description of interventions and their implementation, limit the generalisability of results. These factors restrict the ability to identify specific intervention components that had a positive impact and thus limit opportunities to build evidence and inform future directions. Dietary intervention studies need to clearly describe the intervention trialled, implementation fidelity and process, along with primary and secondary outcome measures and standardised measures. This will then allow the replication and investigation of promising interventions across the diversity of Australian Aboriginal and Torres Strait Islander populations including regional and urban communities where the majority (80\%) live ${ }^{73}$ but where few studies have been conducted. There is a potential for reporting bias in this review where studies have been published in the grey literature or as reports or are currently under review and therefore not included.

National investment is required to prioritise dietary interventions in Australian Aboriginal and Torres Strait Islander communities given the prominence of poor diet as a risk factor for the health inequity suffered by Aboriginal and Torres Strait Islander Australians. ${ }^{8975}$ Improving nutrition is complex, given the extent of barriers to healthy intake in Aboriginal and Torres Strait Islander communities. ${ }^{86974}$ Future efforts should build capacity and evidence informed policy in all food environment settings to support healthy eating, include a focus on urban Aboriginal and Torres Strait Islander communities, ensure strong community engagement and leadership, and a trained and supported Aboriginal and Torres Strait Islander nutrition workforce. The results of this review provide guidance for the next steps to a healthy food future for Australia's first people.

\section{Author affiliations}

${ }^{1}$ Faculty of Health Sciences, University of Sydney, Camperdown, New South Wales, Australia

${ }^{2}$ Charles Perkins Centre, University of Sydney, Camperdown, New South Wales, Australia

${ }^{3}$ Sydney Local Health District, Camperdown, New South Wales, Australia ${ }^{4}$ Department of Sociology, Flinders University, Adelaide, South Australia, Australia ${ }^{5}$ School of Life and Environmental Sciences, University of Sydney, Camperdown, New South Wales, Australia

${ }^{6}$ School of Public Health, University of Queensland, Herston, Queensland, Australia ${ }^{7}$ Department of Nutrition, Dietetics and Food, Monash University, Melbourne, Victoria, Australia

Acknowledgements The authors would like to thank Associate Professor Dr Michael Skilton (University of Sydney, Charles Perkins Centre) for his advice on health outcome data.

Contributors JG, TS, JB, AL and KS contributed to designing the search strategy. TS and KS conducted the searches. TS, KS and JG performed data extractions. $\mathrm{JG}, \mathrm{TS}, \mathrm{JB}, \mathrm{AL}$ and KS contributed to quality assessment. AS quantitatively compared health outcome data. All authors contributed to drafting and revising the manuscript.

Funding The authors have not declared a specific grant for this research from any funding agency in the public, commercial or not-for-profit sectors.

Competing interests None declared. 
Patient consent for publication Not required.

Provenance and peer review Not commissioned; externally peer reviewed.

Data sharing statement The corresponding author has a data which supports Supplementary Table 4 which is not published. Plesae contact the corresponding author and this will be supplied.

Open access This is an open access article distributed in accordance with the Creative Commons Attribution Non Commercial (CC BY-NC 4.0) license, which permits others to distribute, remix, adapt, build upon this work non-commercially, and license their derivative works on different terms, provided the original work is properly cited, appropriate credit is given, any changes made indicated, and the use is non-commercial. See: http://creativecommons.org/licenses/by-nc/4.0/.

\section{REFERENCES}

1. Anderson I, Robson B, Connolly M, et al. Indigenous and tribal peoples' health (The Lancet-Lowitja Institute Global Collaboration): a population study. The Lancet 2016;388:131-57.

2. Australian Institute of Health and Welfare. Australia's Health 2016. Canberra: Australian Institute of Health and Welfare, 2016. https://www.aihw.gov.au/getmedia/9844cefb-7745-4dd8-9ee2f4d1c3d6a727/19787-AH16.pdf.aspx?inline=true. (accessed Jun 2018).

3 Holland C. A Ten-Year Review: The Closing The Gap Strategy And Recommendation. Sydney: Australian Human Rights Commission, 2018. https://www.humanrights.gov.au/sites/default/files/document/ publication/CTG\%202018_FINAL-WEB.pdf. (accessed Jun 2018).

4. Wright P, Lewis P. The Close The Gap Progress And Priorities Report 2017. Sydney: Australian Human Rights Commission, 2017. http:// iaha.com.au/wp-content/uploads/2017/03/Close-the-Gap-report2017.pdf. (accessed Jan 2017).

5. Browne J, Adams K, Atkinson P. Food And Nutrition Programs For Aboriginal And Torres Strait Islander Australians: What Works To Keep People Healthy And Strong? Canberra: Deeble Institute for Health Policy Research, 2016.

6. Department of Health. Implementation Plan For The National Aboriginal And Torres Strait Islander Health Plan 2013-2023. Canberra: Australian Government, 2015.

7. National Health and Medical Research Council. Australian Dietary Guidelines: Providing The Scientific Evidence For Healthier Australian Diets. Canberra: National Health and Medical Research Council, 2013.

8. Australian Bureau of Statistics. National Aboriginal And Torres Strait Islander Health Survey: Consumption Of Food Groups From The Australian Dietary Guidelines, 2012-13. Canberra: Australian Bureau of Statistics, 2016. http://abs.gov.au/ausstats/abs@.nsf/Lookup/ 4727.0.55.008main+features12012-13. (accessed Jan 2017).

9. Australian Bureau of Statistics. National Aboriginal And Torres Strait Islander Health Survey: Nutrition Results - Food And Nutrients, 2012 13. Canberra: Australian Bureau of Statistics, 2015. http://www.abs. gov.au/ausstats/abs@.nsf/Lookup/by\%20Subject/4727.0.55.005 2012-13 Main\%20Features Foods\%20Consumed 4. (accessed Jan 2017).

10. Australian Bureau of Statistics. National Aboriginal And Torres Strait Islander Health Survey: Consumption Of Added Sugars, 2012-13. Canberra: Australian Bureau of Statistics, 2016. http://www.abs.gov. au/ausstats/abs@.nsf/Lookup/by\%20Subject/4727.0.55.009 201213 Main\%20Features Key\%20Findings 1. (accessed Jan 2017).

11. Schembri L, Curran J, Collins L, et al. The effect of nutrition education on nutrition-related health outcomes of Aboriginal and Torres Strait Islander people: a systematic review. Aust N Z J Public Health 2016;40:S42-S47.

12. Browne J, Laurence S, Thorpe S. Acting on food insecurity in urban Aboriginal and Torres Strait Islander communities: policy and practice interventions to improve local access and supply of nutritious food. Australian Indigenous Health/nfoNet 2009 http://www.healthinfonet. ecu.edu.au/health-risks/nutrition/reviews/other-reviews (accessed Nov 2016).

13. Black A. Evidence Of Effective Interventions To Improve The Social And Environmental Factors Impacting On Health: Informing The Development Of Indigenous Community Agreements. Canberra: Office for Aboriginal and Torres Strait Islander Health, 2007.

14. Lee A, Ride K. Review of programs and services to improve Aboriginal and Torres Strait Islander nutrition and food security 2018. Australian Indigenous HealthInfoNet 2018 https://healthinfonet.ecu. edu.au/about/knowledge-exchange-products/.

15. Australian indigenous healthinfoNet. http://www.healthinfonet.ecu. edu.au/ (accessed Dec 2016).
16. Moher D, Liberati A, Tetzlaff J, et al. Preferred reporting items for systematic reviews and meta-analyses: the PRISMA statement. PLoS Med 2009;6:e1000097.

17. National Collaborating Centre for Methods and Tools. Quality Assessment Tool For Quantitative Studies. Hamilton, ON: McMaster University, 2008.

18. National Health and Medical Research Council. NHMRC Additional Levels Of Evidence And Grades For Recommendations For Developers Of Guidelines. Canberra: National Health and Medical Research Council, 2009.

19. Biddle N. Ranking Regions: Revisiting An Index Of Relative Indigenous Socioeconomic Outcomes. Canberra: Australian National University, 2009.

20. Australian Institute of Health and Welfare. Rural, Regional And Remote Health: A Guide To Remoteness Classifications. Canberra: Australian Institute of Health and Welfare, 2004. http://www.aihw.gov. au/publication-detail/?id=6442467589. (accessed Apr 2016).

21. National Health and Medical Research Council. Ethical conduct in research with Aboriginal and Torres Strait Islander Peoples and communities: Guidelines for researchers and stakeholders. Canberra: National Health and Medical Research Council, 2018.

22. Liberato SC, Brimblecombe J, Ritchie J, et al. Measuring capacity building in communities: a review of the literature. BMC Public Health 2011;11:850.

23. Gwynn J, Lock M, Turner N, et al. Aboriginal and Torres Strait Islander community governance of health research: Turning principles into practice. Aust J Rural Health 2015;23:235-42.

24. National Health and Medical Research Council. The NHMRC Road Map II: A Strategic Framework For Improving The Health Of Aboriginal And Torres Strait Islander People Through Research. Canberra: National Health and Medical Research Council, 2010.

25. National Health and Medical Research Council. Keeping Research On Track: A Guide For Aboriginal And Torres Strait Islander Peoples About Health Research Ethics. Canberra: National Health and Medical Research Council, 2005.

26. Jamieson LM, Paradies YC, Eades S, et al. Ten principles relevant to health research among Indigenous Australian populations. Med $J$ Aust 2012;197:16-18.

27. World Cancer Research Fund International. NOURISHING - Our Policy Framework To Promote Healthy Diets And Reduce Obesity. London: World Cancer Research Fund International, 2016. https:// www.wcrf.org/int/policy/nourishing/our-policy-framework-promotehealthy-diets-reduce-obesity. (accessed Feb 2018).

28. Cargo M, Marks E, Brimblecombe J, et al. Integrating an ecological approach into an Aboriginal community-based chronic disease prevention program: a longitudinal process evaluation. BMC Public Health 2011;11:299.

29. Rowley K, Doyle J, Johnston L, et al. Strengths and limitations of a tool for monitoring and evaluating First Peoples' health promotion from an ecological perspective. BMC Public Health 2015;15:1215.

30. Davis CE, Gordon D, LaRosa J, et al. Correlations of plasma highdensity lipoprotein cholesterol levels with other plasma lipid and lipoprotein concentrations. Circulation 1980;62:IV24-30.

31. Cohen J. The earth is round $(p<.05)$. Am Psychol 1994;49:997-1003.

32. Livshits G, Malkin I, Williams FM, et al. Longitudinal study of variation in body mass index in middle-aged UK females. Age 2012;34:1285-94.

33. Bookstein L, Gidding SS, Donovan M, et al. Day-to-day variability of serum cholesterol, triglyceride, and high-density lipoprotein cholesterol levels. Impact on the assessment of risk according to the National Cholesterol Education Program guidelines. Arch Intern Med 1990;150:1653-7.

34. Coyne T, Dowling M, Condon-Paoloni D. Evaluation of preschool meals programmes on the nutritional health of Aboriginal children. Med J Aust 1980;2:369-75.

35. Quinn E, O'Hara BJ, Ahmed N, et al. Enhancing the get healthy information and coaching service for Aboriginal adults: evaluation of the process and impact of the program. Int $J$ Equity Health 2017;16:168.

36. Brimblecombe J, Ferguson M, Chatfield MD, et al. Effect of a price discount and consumer education strategy on food and beverage purchases in remote Indigenous Australia: a stepped-wedge randomised controlled trial. Lancet Public Health 2017;2:e82-95.

37. McMahon E, Webster J, Brimblecombe J. Effect of $25 \%$ sodium reduction on sales of a top-selling bread in remote indigenous australian community stores: a controlled intervention trial. Nutrients 2017;9:214.

38. Lee AJ, Bailey AP, Yarmirr D, et al. Survival tucker: improved diet and health indicators in an aboriginal community. Aust J Public Health 1994;18:277-85. 
39. Rowley KG, Daniel M, Skinner K, et al. Effectiveness of a communitydirected 'healthy lifestyle' program in a remote Australian aboriginal community. Aust N Z J Public Health 2000;24:136-44.

40. Scrimgeour D, Rowse T, Knight S. Food-purchasing behaviour in an Aboriginal community. 2. Evaluation of an intervention aimed at children. Aust J Public Health 1994;18:67-70.

41. Jones R, Smith F. Are there health benefits from improving basic nutrition in a remote Aboriginal community? Aust Fam Physician 2006;35:453-4.

42. O'Dea K, Sinclair AJ. Increased proportion of arachidonic acid in plasma lipids after 2 weeks on a diet of tropical seafood. Am J Clin Nutr 1982;36:868-72.

43. O'Dea K. Marked improvement in carbohydrate and lipid metabolism in diabetic Australian aborigines after temporary reversion to traditional lifestyle. Diabetes 1984;33:596-603.

44. Rowley KG, Su Q, Cincotta M, et al. Improvements in circulating cholesterol, antioxidants, and homocysteine after dietary intervention in an Australian Aboriginal community. Am J Clin Nutr 2001;74:442-8.

45. Rowley KG, Lee AJ, Yarmirr D, et al. Homocysteine concentrations lowered following dietary intervention in an aboriginal community. Asia Pac J Clin Nutr 2003;12:92-5.

46. Lee AJ, Bonson AP, Yarmirr D, et al. Sustainability of a successful health and nutrition program in a remote aboriginal community. Med $J$ Aust 1995;162:632-5.

47. O'Dea K, Spargo RM. Metabolic adaptation to a low carbohydratehigh protein ('traditional') diet in Australian Aborigines. Diabetologia 1982;23:494-8.

48. O'Dea K, Sinclair AJ. The effects of low-fat diets rich in arachidonic acid on the composition of plasma fatty acids and bleeding time in Australian aborigines. J Nutr Sci Vitaminol 1985;31:441-53.

49. O'Dea K, Naughton JM, Sinclair AJ, et al. Lifestyle change and nutritional status in kimberley aborigines. Aust Aborig Stud 1987;1:46-51.

50. McDermott R, Rowley KG, Lee AJ, et al. Increase in prevalence of obesity and diabetes and decrease in plasma cholesterol in a central Australian aboriginal community. Med J Aust 2000;172:480-4.

51. Jones R, Smith F. Fighting disease with fruit. Aust Fam Physician 2007;36:863-4.

52. Chan LC, Ware R, Kesting J, et al. Short term efficacy of a lifestyle intervention programme on cardiovascular health outcome in overweight Indigenous Australians with and without type 2 diabetes mellitus. The healthy lifestyle programme (HELP). Diabetes Res Clin Pract 2007;75:65-71.

53. Pettigrew S, Jongenelis $\mathrm{MI}$, Moore $\mathrm{S}$, et al. A comparison of the effectiveness of an adult nutrition education program for Aboriginal and non-Aboriginal Australians. Soc Sci Med 2015;145:120-4.

54. McDonald B. Why mepla no kaikai whiteman food? Because store no got. Aust Nurses J 1992;21:16-19.

55. Brimblecombe JK, McDonnell J, Barnes A, et al. Impact of income management on store sales in the Northern Territory. Med J Aust 2010;192:549-54.

56. Butler R, Tapsell L, Lyons-Wall P. Trends in purchasing patterns of sugar-sweetened water-based beverages in a remote Aboriginal community store following the implementation of a communitydeveloped store nutrition policy. Nutrition \& Dietetics 2011;68:115-9.

57. Lee A, Bonson AP, Powers JR. The effect of retail store managers on aboriginal diet in remote communities. Aust N Z J Public Health 1996;20:212-4.
58. Lee AJ, Hobson V, Katarski L. Review of the nutrition policy of the Arnhem Land Progress Association. Aust N Z J Public Health 1996;20:538-44.

59. Reilly RE, Cincotta M, Doyle J, et al. A pilot study of Aboriginal health promotion from an ecological perspective. BMC Public Health 2011;11:749.

60. Ferguson M, O'Dea K, Holden S, et al. Food and beverage price discounts to improve health in remote Aboriginal communities: mixed method evaluation of a natural experiment. Aust N Z J Public Health 2017;41:32-7.

61. Brimblecombe J, Bailie R, van den Boogaard C, et al. Feasibility of a novel participatory multi-sector continuous improvement approach to enhance food security in remote Indigenous Australian communities. SSM Popul Health 2017;3:566-76.

62. Egger $\mathrm{G}$, Fisher $\mathrm{G}$, Piers $\mathrm{S}$, et al. Abdominal obesity reduction in indigenous men. Int J Obes Relat Metab Disord 1999;23:564-9.

63. Gracey M, Bridge E, Martin D, et al. An Aboriginal-driven program to prevent, control and manage nutrition-related "lifestyle" diseases including diabetes. Asia Pac J Clin Nutr 2006;15:178-88.

64. Longstreet D, Heath D, Savage I, et al. Estimated nutrient intake of urban Indigenous participants enrolled in a lifestyle intervention program. Nutrition \& Dietetics 2008;65:128-33.

65. Rowley KG, Gault A, McDermott R, et al. Reduced prevalence of impaired glucose tolerance and no change in prevalence of diabetes despite increasing BMI among Aboriginal people from a group of remote homeland communities. Diabetes Care 2000;23:898-904.

66. O'Dea K, Spargo RM, Akerman K. The effect of transition from traditional to urban life-style on the insulin secretory response in Australian Aborigines. Diabetes Care 1980;3:31-7.

67. Black AP, Vally $H$, Morris $P$, et al. Nutritional impacts of a fruit and vegetable subsidy programme for disadvantaged Australian Aboriginal children. Br J Nutr 2013;110:2309-17.

68. Black AP, Vally H, Morris PS, et al. Health outcomes of a subsidised fruit and vegetable program for Aboriginal children in northern New South Wales. Med J Aust 2013;199:46-50.

69. Wolfenden L, Jones J, Williams CM, et al. Strategies to improve the implementation of healthy eating, physical activity and obesity prevention policies, practices or programmes within childcare services. Cochrane Database Syst Rev 2016;10:CD011779.

70. Magnus A, Cobiac L, Brimblecombe J, et al. The cost-effectiveness of a $20 \%$ price discount on fruit, vegetables, diet drinks and water, trialled in remote Australia to improve Indigenous health. PLoS One 2018;13.

71. Swinburn B, Wood A. Progress on obesity prevention over 20 years in Australia and New Zealand. Obes Rev 2013;14 Suppl 2:S60-8.

72. Anderson I, Crengle S, Kamaka ML, et al. Indigenous health in Australia, New Zealand, and the Pacific. Lancet 2006;367:1775-85.

73. Australian Institute of Health and Welfare. The Health And Welfare Of Australia's Aboriginal And Torres Strait Islander Peoples: 2015. Canberra: Australian Institute of Health and Welfare, 2015. http:// www.aihw.gov.au/WorkArea/DownloadAsset.aspx?id=60129551281. (accessed Nov 2016).

74. Kettings C, Sinclair AJ, Voevodin M. A healthy diet consistent with Australian health recommendations is too expensive for welfaredependent families. Aust N Z J Public Health 2009;33:566-72.

75. Australian Institute of Health and Welfare. Australian Burden Of Disease Study: Impact And Causes Of IIIness And Death In Aboriginal And Torres Strait Islander People 2011. Canberra: Australian Institute of Health and Welfare, 2016. 